\title{
Stokes parameters in undergraduate laboratory exercises
}

\section{Gregory Topasna, Daniela Topasna}

Gregory A. Topasna, Daniela M. Topasna, "Stokes parameters in undergraduate laboratory exercises," Proc. SPIE 9666, 11th Education and Training in Optics and Photonics Conference, 96660R (5 June 2009); doi: $10.1117 / 12.2207977$

SPIE Event: Eleventh International Topical Meeting on Education and Training in Optics and Photonics, 2009, St. Asaph, United Kingdom 


\title{
Stokes parameters in undergraduate laboratory exercises
}

\author{
Gregory A. Topasna and Daniela M. Topasna \\ Department of Physics and Astronomy \\ Virginia Military Institute, Lexington, VA
}

\begin{abstract}
Polarization is a concept most students readily understand in terms of the preferential direction of electric field vectors. The visualization of the electric field component of an electromagnetic wave facilitates the understanding of a large body of knowledge concerning propagation and measurement of completely and partially polarized light. Little known to undergraduate students, however, is the Stokes parameters and students typically receive a cursory treatment regarding their usefulness in describing and measuring polarized light in a laboratory or astronomical setting. We present laboratory exercises where students use Stokes parameters when measuring and describing the polarization of electromagnetic radiation and in the statistical analysis of polarized light.
\end{abstract}

Keyword list: polarized light, Stokes parameters, numerical modeling

\section{INTRODUCTION}

Most students in science and engineering are introduced to polarization by the preferential alignment of the electric field component of electromagnetic radiation (presumably, after having passed through a polarizer or having been partially polarized upon reflection). In advanced classes further concepts are explored such as birefringence, optical activity, dichroism, and scattering and the mathematical approach to polarization is through the Stokes parameters, the Jones vectors, and Jones and Mueller matrices ${ }^{1,2}$. As thorough as this introduction usually is, students are usually left with few advanced labs where they can explore some of the topics mentioned above. In particular, students may never perform a laboratory exercise where they are required to determine the state of polarization of light in various settings. Likewise, how to correctly handle polarization statistics is requisite knowledge that all students should have an exposure to.

Eleventh International Topical Meeting on Education and Training in Optics and 
The lab we introduce remedies this situation because it requires students to determine the state of polarization (degree of polarization and position angle) of laser light and how to correctly handle polarization statistics. Additionally, the students gain valuable skill and understanding about optics and optical components (such as the half-wave plate, Wollaston prism, telescope, and CCD cameras) because they are required to set-up and align the experiment themselves.

\section{BACKGROUND}

The polarization state of radiation is given by the Stokes parameters $I, Q, U$, and $V$ which are defined in terms of the amplitude of electric field components of orthogonal states along two distinct axes perpendicular to the direction of propagation. For example, by choosing the polarization of a simple wave such that $E_{r 0}$ and $E_{l 0}$ are the respective perpendicular and parallel amplitude components in a plane perpendicular to the direction of propagation, the Stokes parameters describing the polarization of this wave are given by

$$
\begin{aligned}
& I=E_{l 0}^{2}+E_{r 0}^{2}=\sqrt{Q^{2}+U^{2}+V^{2}} \\
& Q=E_{l 0}^{2}-E_{r 0}^{2}=I \cos 2 \beta \cos 2 \theta \\
& U=-2 E_{l 0} E_{r 0} \cos \left(\varepsilon_{l}-\varepsilon_{r}\right)=I \cos 2 \beta \sin 2 \theta \\
& V=2 E_{l 0} E_{r 0} \sin \left(\varepsilon_{l}-\varepsilon_{r}\right)=I \sin 2 \beta
\end{aligned}
$$

where $\varepsilon_{l}$ and $\varepsilon_{r}$ are constants, $\theta$ is the angle the long axis of the polarization ellipse makes with respect to the direction $I$ and $\beta$ is the angle whose tangent is the ratio of the two axes of the polarization ellipse. ${ }^{3}$ While a more in depth treatment of polarization described above can readily be found in advanced texts ${ }^{4}$, it is the properties of the Stokes parameters that we are more interested in, and in particular, the properties of linearly polarized radiation (where $V=0$ ).

For plane polarized light, the degree of partial polarization is given by the expression

$p=\sqrt{Q^{2}+U^{2}} / I$ which can be written in terms of the normalized Stokes parameters $(q=Q / I$, and $u=U / I)$ as

$$
p=\sqrt{q^{2}+u^{2}} .
$$

By decomposing the partially polarized light into two beams that are completely polarized, we can define and $I_{\text {min }}$ and $I_{\max }$ that recasts the Stokes parameters as ${ }^{3}$ 


$$
\begin{aligned}
& I=I_{\text {max }}+I_{\text {min }} \\
& Q=\left(I_{\text {max }}-I_{\text {min }}\right) \cos 2 \theta \\
& U=\left(I_{\text {max }}-I_{\text {min }}\right) \sin 2 \theta
\end{aligned}
$$

from which the polarization position angle can be obtained as

$$
\tan (2 \theta)=\frac{Q}{U}=\frac{q}{u}
$$

It only leaves us now to determine $I_{\min }$ and $I_{\max }$ which can be accomplished in a number of ways. One way is to pass partially polarized light through an ideal linear polarizer and measure the transmitted intensity at four angles $\left(0^{\circ}, 45^{\circ}, 90^{\circ}\right.$, and $\left.135^{\circ}\right)$. The normalized Stokes parameters would be

$$
q=\frac{Q}{I}=\frac{I\left(0^{\circ}\right)-I\left(90^{\circ}\right)}{I\left(0^{\circ}\right)+I\left(90^{\circ}\right)} \quad u=\frac{U}{I}=\frac{I\left(45^{\circ}\right)-I\left(135^{\circ}\right)}{I\left(45^{\circ}\right)+I\left(135^{\circ}\right)}
$$

In the laboratory exercise we describe below, polarized light is passed through a half-wave plate and then a Wollaston prism which splits the one beam into two distinct, mutually orthogonal polarization states. The total intensity is given by the sum of the intensities of the two beams, which is the I Stokes parameter, and the difference in intensity is the $Q$ Stokes parameter. If the fast-axis of the half-wave plate is then rotated by $22.5^{\circ}$, the difference in intensity between the two beams is the $U$ Stokes parameter. By using a half-wave plate, the Stokes parameters are obtained by a single rotation and measurement of the two intensities at each rotation. Specifically, the Stokes parameters are

$$
q=\frac{Q}{I}=\frac{I_{e}\left(0^{\circ}\right)-I_{o}\left(0^{\circ}\right)}{I_{e}\left(0^{\circ}\right)+I_{o}\left(0^{\circ}\right)} \quad u=\frac{U}{I}=\frac{I_{e}\left(22.5^{\circ}\right)-I_{o}\left(22.5^{\circ}\right)}{I_{e}\left(22.5^{\circ}\right)+I_{o}\left(22.5^{\circ}\right)}
$$

where $I_{o}$ is the intensity of the ordinary ray and $I_{e}$ is the intensity of the extraordinary ray measured at the half-wave plate position angles indicated. The half-wave plate can be further rotated in $22.5^{\circ}$ increments for subsequent measurements of $I, Q$, and $U$ (only the position angle respect to a fiduciary setting needs to be accounted for).

\section{EXPERIMENTAL SETUP}

The layout of the optical components is shown in Figure 1. The system is a two-beam device in that light from the laser forms two spots on the screen (S) after leaving the Wollaston prism (WP). The laser used is a Pasco

diode laser that produces a slightly elliptical shaped beam and the aperture (A) serves to help make the beam 
circular. The neutral density filter (ND) attenuates the light so that variable integration times of the ChargeCoupled Device (CCD) are possible. The polarizer (P1) sets the position angle to be detected. (An additional polarizer can be inserted after this polarizer, P2 in the diagram below, to adjust the intensity as well as observe the Law of Malus). The polarizers are mounted in rotatable stages that can be set to an accuracy of $\leq 1^{\circ}$. Since the beam has a fairly large diameter, a telescope composed of two plano-convex lenses (L1) and (L2) are used to reduce the diameter of the beam by a factor of $\sim 10$. The analyzer is the half-wave plate (HWP) in a rotatable stage with and the Wollaston prism (WP) has a $20^{\circ}$ angle of separation between the two emerging beams. The image of the two spots on the screen $(S)$ is obtained using the CCD camera with lens L3 which is an $\mathrm{f} / 1.8$ Nikon camera lens.

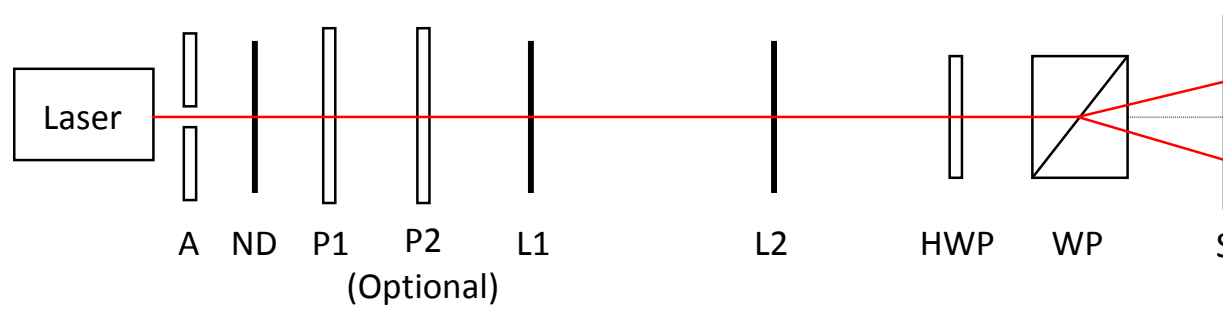

Figure 1. Layout of the optical components.

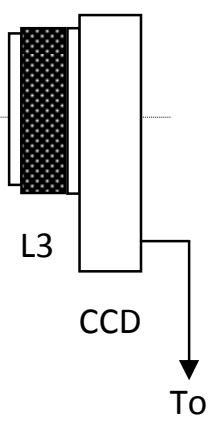

Computer

Figure 2 shows the arrangement of the components on the breadboard for a completed setup and Figure 3 is a close up view of the two spots on the screen with the Wollaston prism and half-wave plate seen on the left. Barely discernable in Figure 3 is the image of the central beam (i.e. the laser beam that has not split into the ordinary and extraordinary rays).

The student starts out by examining Figure 1 and selecting the necessary components needed to perform the exercise. Having learned how to mount components in holders and on the breadboard he precedes to layout the components in the general area where they will be securely mounted. One-by-one he aligns the optical components so that the central beam is in the middle of the camera lens (installing the Wollaston prism and the screen last). The student is asked to build a telescope to reduce the size of the beam by a factor of $\sim 10$ and the choice of lenses is left to him. In doing this, the student becomes a contributor in the design process which helps him to take psychological ownership of the project. 


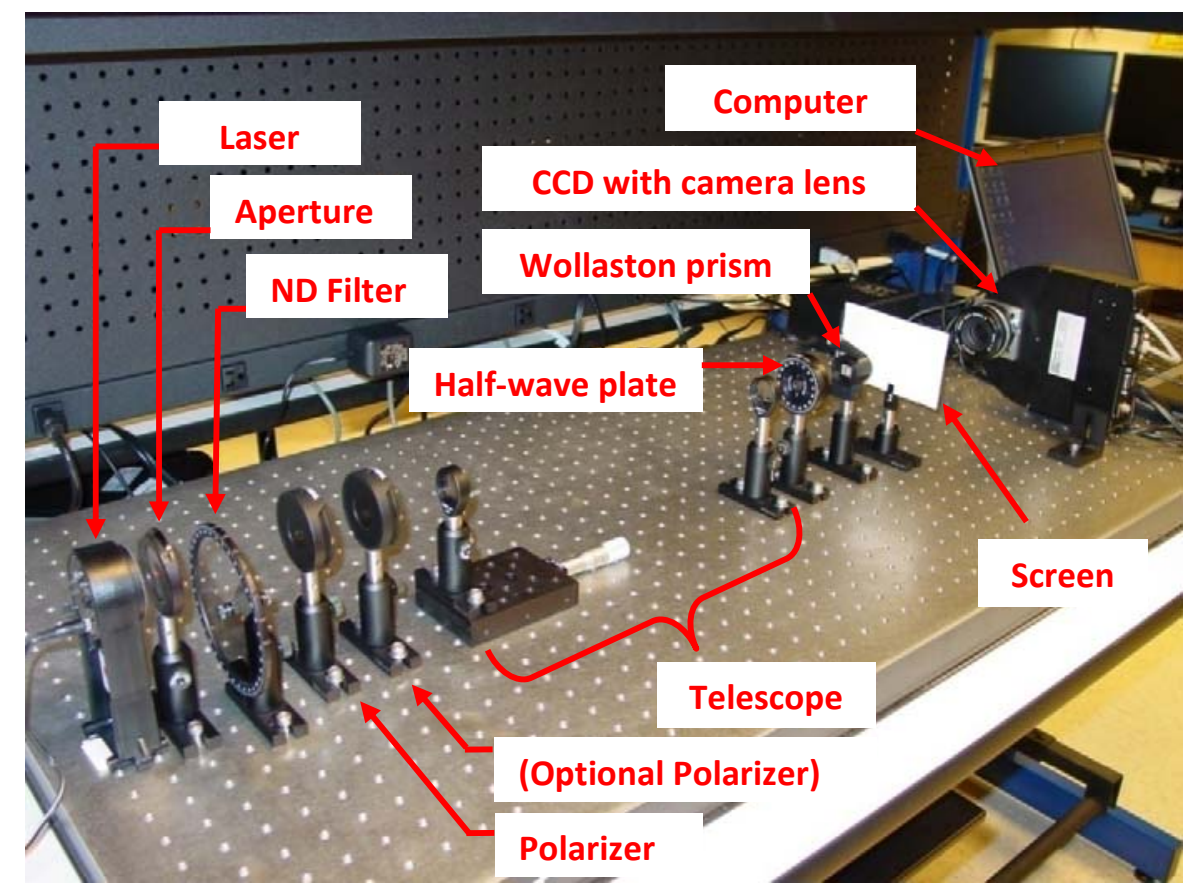

Figure 2. Physical arrangement of components.

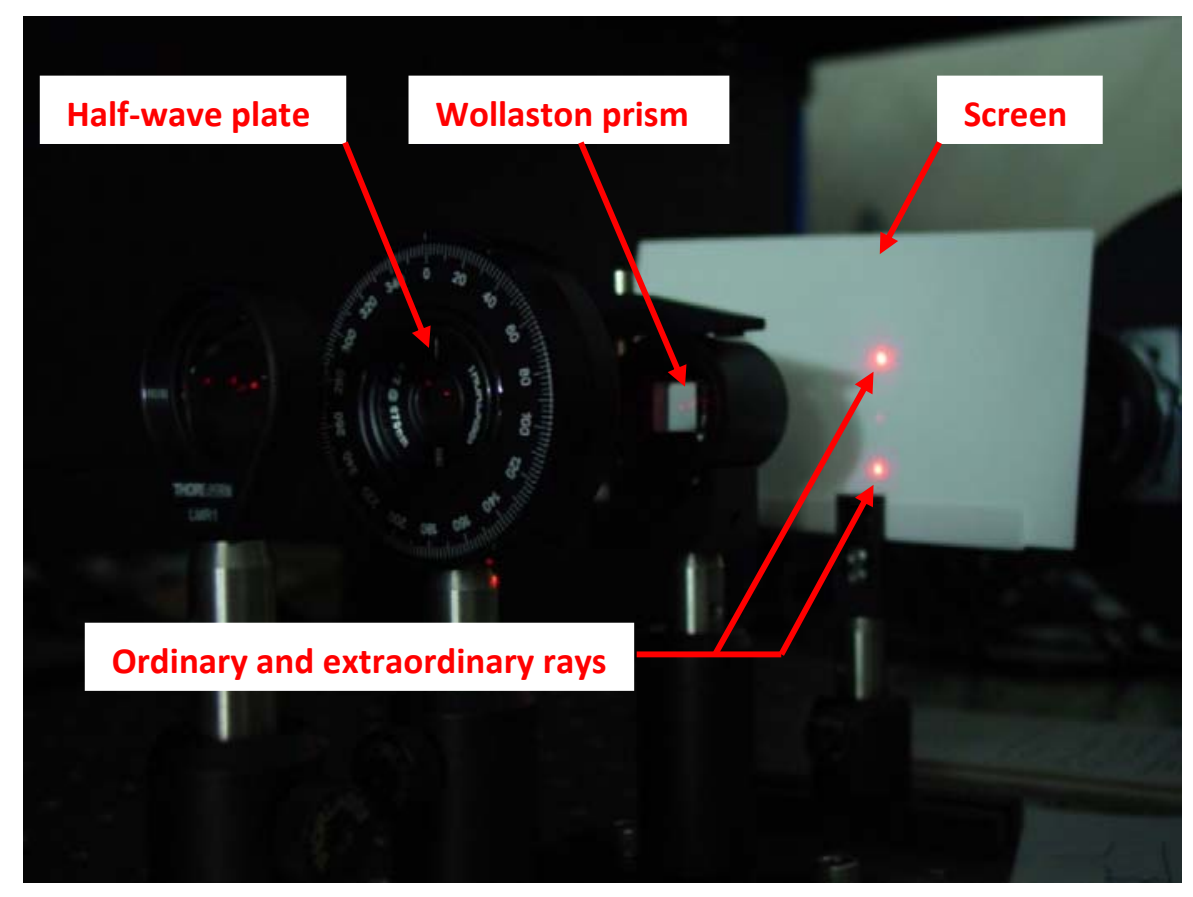

Figure 3. Image of the two spots on the screen after emerging from the Wollaston prism (left of the screen). The half-wave plate is in a rotatable stage to the left of the Wollaston prism. 
Once the layout and alignment is complete and checked by both the student and the instructor, the student is instructed to examine his setup more closely. Using a piece of Polaroid with a marked transmission axis, he examines the beam between the optical elements to ascertain if any polarization is present and what the position angle might be. In the case of the Pasco laser used her, he finds that the beam is polarized to a high degree and easily determines the position angle. He follows the beam with the Polaroid, past the polarizers and telescope, and observes the effect the half-wave plate has as it is rotated. Finally, he sets the fast axis of the half-wave plate vertical and observes the polarization state of the two beams emerging from the Wollaston prism in order to determine their nature. With the Wollaston prism oriented to produce the two spots as shown in Figure 3, the top beam is polarized horizontally with respect to the optical table and the bottom beam is polarized perpendicular to the table.

Since the two beams are the ordinary and extraordinary rays, Figure 4 shows the behavior of the two spots as the half-wave plate is rotated from $0^{\circ}$ to $157.5^{\circ}$ in increments of $22.5^{\circ}$. The left spot in Figure 4 is the top, horizontally polarized beam shown in Figure 3 while the right spot is the lower, vertically polarized beam. As the half-wave plate is rotated to $180^{\circ}$ this corresponds naturally to $0^{\circ}$ again and the pattern repeats itself. For purposes of identifying how far the half-wave plate rotates, the measurements obtain until $337.5^{\circ}$ at every $22.5^{\circ}$ is recorded at the angle it was made.
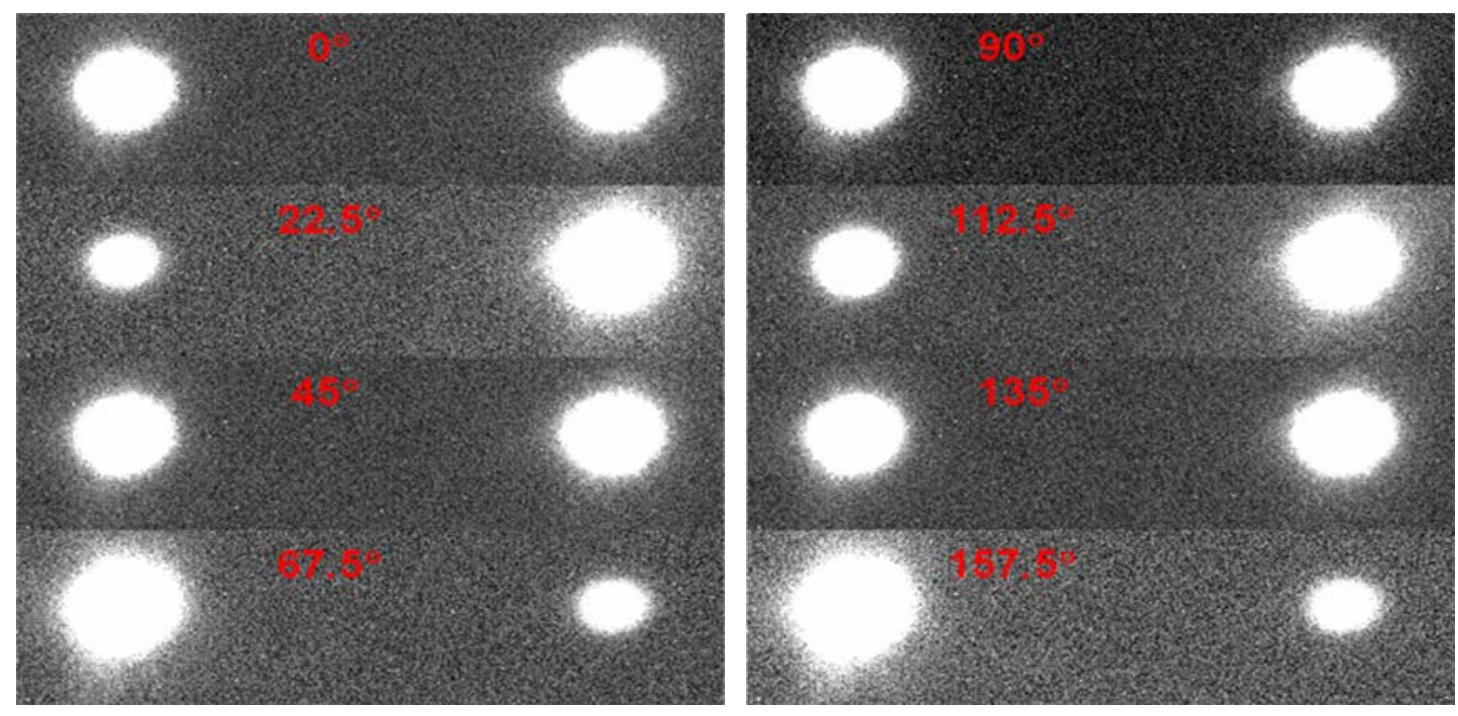

Figure 4. Image of the two spots on the screen. The left spot is horizontally polarized with respect to the optical table while the right beam is vertically polarized with respect to the table. 
In order to measure the intensity of each spot, aperture photometry is performed using the software program Mira Pro by Mirametrics ${ }^{5}$. This involves setting the ON aperture to measure the amount of counts (or ADU Analog Digital Units) for each of the two spots and subtracting a background (the "OFF") that has the same area as the ON. The median background is obtained using an annulus surrounding the ON aperture that has an inner radius larger than the ON radius with a typical width on the order of 10 to 20 pixels.

In order to determine an appropriate size of the ON aperture, the student first plots the signal (ON - OFF) in a series of increasing apertures centered on the centroid of one of the spots as a function of aperture radius. Additionally, the signal-to-noise $(\mathrm{S} / \mathrm{N})$ ratio is plotted for each of the radii as well. As the size of the aperture increases, the signal will increase as will the $\mathrm{S} / \mathrm{N}$ ratio. The signal will start to level off as the aperture increases beyond where the signal is indistinguishable from the background noise. This simply reflects the fact that the subtracted background is approximately equal to the signal added by the increased aperture size. However, as the aperture is increased beyond a certain point, the $\mathrm{S} / \mathrm{N}$ ratio will start to decline as the aperture becomes too large.

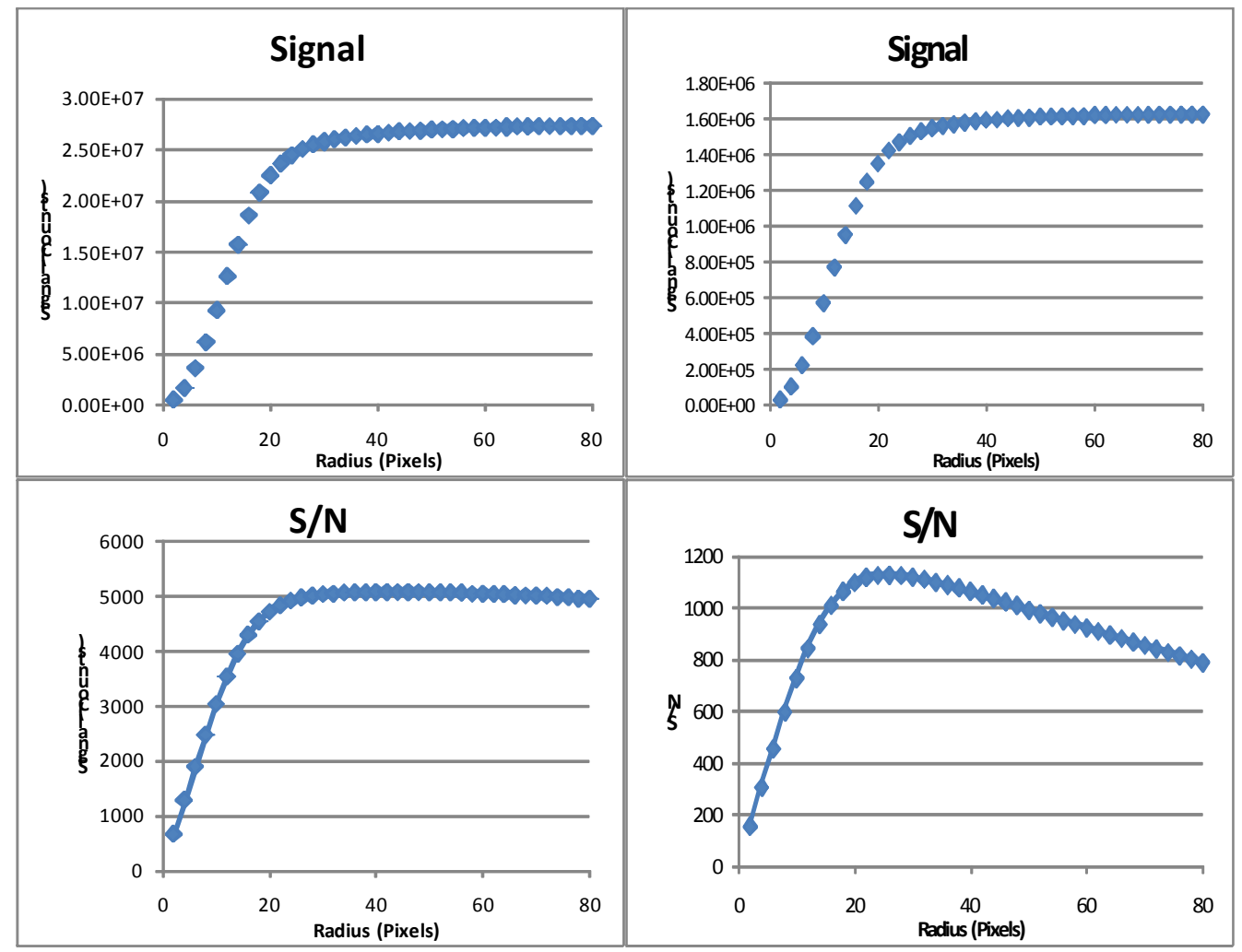

Figure 5. Signal (top) and $\mathrm{S} / \mathrm{N}$ ratio (bottom) as a function of $\mathrm{ON}$ aperture size measured in pixels for a bright spot (left) and dim spot (right). 
Figure 5 illustrates how the signal-to-noise ratio changes for a bright spot (left graphs) and a dim spot (right graphs). While the signal-to-noise ratio may decrease more rapidly for the dim spot, the actual S/N ratio itself remains quite high. By choosing an aperture radius that is larger than where the $\mathrm{S} / \mathrm{N}$ ratio peaks, the student is assured of accurately measuring all of the light from both spots using a single size aperture without significant loss of signal.

\section{MEASUREMENTS}

In order to measure polarization, polarizer $\mathrm{P} 1$ is set to a known angle. The neutral density filter should be initially set so that light from the laser does not saturate the CCD. The half-wave plate is rotated by $22.5^{\circ}$ increments from the fiduciary setting of $0^{\circ}$ with 10 images taken at each angle. Aperture photometry (described above) is performed on each spot in each image in order to determine the intensity of the ordinary $I_{o}$ and extraordinary $I_{e}$ ray. From these measurements the normalized Stokes parameters are computed using equations (5). Given the high degree of polarization and large signal-to-noise ratio, it would be acceptable to estimate the degree of polarization and position angle for each pair of measurements and average these values. However, in general, when the degree of polarization is low and/or the signal-to-noise ratio is small, one should not average the individual polarization measurements, rather, the average of the Stokes parameters is acceptable since they are normally distributed. Once the averages of $q$ and $u$ are determined, the degree of polarization and position angle are then computed.

The measurements from one experiment are shown in Table 1. The data show that there is an instrumental polarization of about $1^{\circ}$ mostly likely caused by a misalignment of an optical element(s) but is otherwise in agreement with the set polarizer angle. Figure 6 demonstrates the agreement between the measured polarization and set polarizer angle. Students can estimate the error in the position angle using standard techniques in error propagation and their measurements of $u$ and $q$. since they are nearly normally distributed. Since the position angle is given by equation 1.4 , calculation of the error in position angle $(\delta \theta)$ is

$$
\delta \theta=\frac{1}{2} \frac{u \sigma_{\bar{q}}-q \sigma_{\bar{u}}}{u^{2}+q^{2}}
$$

where average values and normal error statistics are used. 
The measured degree of polarization was consistently around $99 \%$ owing to the polarized output of the laser. Since the degree of polarization is very large in this exercise, no polarization estimator is necessary.

However, for the sake of completeness, students calculate an estimation of the polarization as if it were small. When the degree of estimator is necessary due

Table 1 Experimental Results

polarization is small, an

to the nature of polarization

Angle of P2 Measured Angle Uncertainty

(Degrees) (Degrees) (Degrees)

\begin{tabular}{|c|c|c|}
\hline \hline $0^{\circ}$ & -1.06 & 0.06 \\
\hline $20^{\circ}$ & 19.3 & 0.04 \\
\hline $40^{\circ}$ & 38.4 & 0.01 \\
\hline $60^{\circ}$ & 58.8 & 0.02 \\
\hline $80^{\circ}$ & 78.7 & 0.7 \\
\hline $100^{\circ}$ & 99.1 & 1.0 \\
\hline $120^{\circ}$ & 118.7 & 0.05 \\
\hline $140^{\circ}$ & 138.6 & 0.5 \\
\hline $160^{\circ}$ & 158.9 & 0.06 \\
\hline
\end{tabular}

statistics $^{6,7}$ and the one used here is the Wardle estimator ${ }^{8}$ given by $\hat{p}=\sqrt{p^{2}-\sigma_{p}^{2}}$ where $p$ is the measured polarization and $\sigma_{p}$ the uncertainty in the measured polarization. The uncertainty can be estimated in various ways ${ }^{9}$ however, alignment of the polarizer and wave plate are most likely the largest sources of error. Therefore, a realistic estimate of $\sigma_{p}$ is given by Serkowski as $\sigma_{p}=p \cdot \sigma_{\varphi}$ where in this exercise we take $\sigma_{\varphi}$ to be the uncertainty in the alignment of the optics expressed in radians. Since the half-wave plate is rotated by hand and read from a rotation stage, an estimate of $\sigma_{\varphi}$ is on the order of $\sim 1^{\circ}$ so the estimated uncertainty in polarization $\sigma_{p}$ is $\sim 0.02$ therefore making $\hat{p} \square p$.

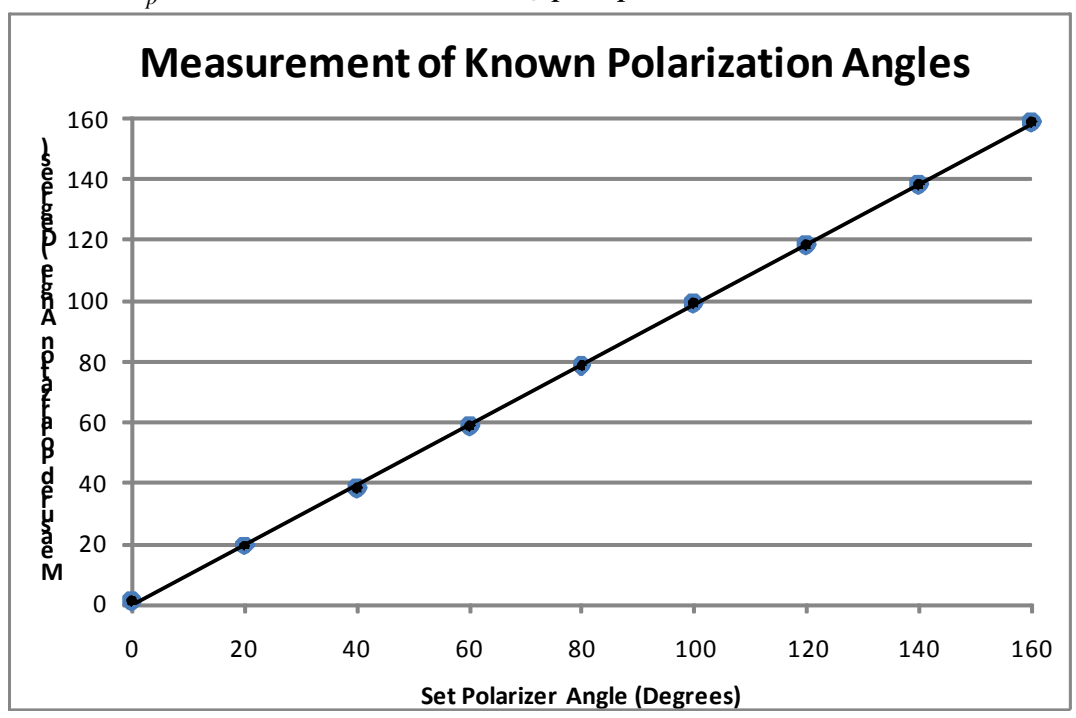

Figure 6. Measurements of the measured polarization position angle as a function of the set polarizer angle. 


\section{DISCUSSION}

This exercise bridges a gap between theory and practice by familiarizing students with Stokes parameters and having them measure polarization using a Stokes parameter technique. The students improve their optics skills by setting up the experiment on an optical bench, calculating distances for the telescope used, and integrating the various components. They acquire knowledge of CCD operation as well as photometry and learn the basics of handling polarization statistics. There is also room for innovation and improvement that students are encouraged to contemplate. For instance, the introduction of a second polarizer in the set up could be used to vary the intensity of the beam and observe the Law of Malus. This is left as an exercise for students to suggest and perform if they wish.

The cost of the experiment can be nominal if various components are readily available in the lab. The CCD and computer software used to acquire and measure the light intensity is a costly part of the experiment and alternative ways of measuring the intensity of the two beams can be explored. The cost of the wave-plate and Wollaston prism can be substantial, but it is assumed that these are found in most optics laboratories.

\section{REFERENCES}

[1] Pedrotti, Frank L., S.J. and Pedrotti, Leno S., "Introduction to optics", Prentice Hall, $2^{\text {nd }}$ edition, 1993.

[2] Hecht, E., "Optics", Addison-Wesley, $3^{\text {rd }}$ edition, 1998.

[3] Serkowsi, K., "Polarization of Starlight" (In "Advances in Astronomy and Astrophysics", Volume 1) Kopal, Zdenek, Ed., Academic Press, New York, 1962.

[4] Jackson, J. D., "Classical Electrodynamics", 2 ${ }^{\text {nd }}$ Edition, John Wiley \& Sons, New York, 1975.

[5] http://www.mirametrics.com/

[6] Clarke, D., Schwarz, H. E., and Stewart, B. G., "Astronomy and Astrophysics", 145, 232, 1983

[7] Simmons, J. F. L. and Stewart, B. G., "Astronomy and Astrophysics", 142, 100, 1985

[8] Wardle, J. F. C. and Kronberg, P. P., "The Astrophysical Journal”, 194, 249, 1974

[9] Clarke, D. and Stewart, B. G., "Vistas in Astronomy", 29, 27, 1986

\section{ACKNOWLEDGEMENTS}

The authors would like to acknowledge the travel support from the Virginia Military Institute's Jackson Hope Fund and National Academy of Sciences (NAS). 